\title{
Advances in respiratory medicine
}

\author{
Anne E Tattersfield
}

Anne E Tattersfield OBE MD FRCP FMedSci, Emeritus Professor of Respiratory Medicine, Nottingham City Hospital Clin Med $2007 ; 7: 442-9$

\section{Introduction}

Respiratory medicine was undergoing major changes when I entered the specialty in the mid-1960s. Tuberculosis (TB) was in decline, almost in freefall, and respiratory physiology was enjoying a golden era. We were able to measure lung function in a broad range of patients, and use the findings to guide management. Cohort studies of smokers and occupational workers were starting to provide data on the causes and natural history of conditions such as chronic bronchitis and lung cancer. The Medical Research Council trials for the treatment of respiratory diseases - anti-TB chemotherapy for TB and cortisone acetate for asthma - were some of the earliest randomised clinical trials. The potential benefit from basing treatment on a more secure footing was obvious.

The organisation of chest medicine was also undergoing major development. The overwhelming workload from patients with TB earlier in the century had meant that for many physicians inpatient work was carried out in sanatoria, and outpatient work in chest clinics. As the prevalence of TB declined, the beds moved into general hospitals and chest physicians undertook acute general medicine. Outpatient work continued for sometime in chest clinics where a dedicated chest X-ray service was available. Chest physicians would report on the $\mathrm{X}$-rays at the end of the clinic and call patients back for further investigation or treatment as appropriate. The service provided rapid access and continuity of care and was highly efficient, with notes and X-rays kept in the clinic.

I have little recollection of being taught respiratory medicine as a medical student so my earliest memories relate to my junior hospital posts in Newcastle, Leicester and London (Central Middlesex, the Brompton, Hammersmith and the London) between 1964 and 1974. At this time there was more variation in hospital practice and working in several hospitals ensured wide and varied experience. For the most part, the patients we saw had similar illnesses to those seen today - pneumonia, lung cancer, asthma, bronchiectasis, TB, sarcoid and interstitial lung diseases. Chronic obstructive pulmonary disease (COPD) was common, though called chronic bronchitis and emphysema. Some of today's diseases were not seen or not recognised, including AIDS, adult respiratory distress syndrome and sleep apnoea. Opportunistic infections were rare and outside specialist centres we saw little cystic fibrosis (CF) since few patients survived beyond adolescence.

Respiratory physicians often worked closely with cardiologists and some physicians covered both specialties. It was obvious that cardiology had forged ahead once cardiac catheterisation was introduced and a similar sea change in respiratory medicine was anticipated as the ability to investigate patients increased. Lung function testing did not cause quite the revolution that cardiac catheterisation achieved but a more gradual revolution occurred as other means of investigating lung diseases were introduced.

\section{Investigative techniques}

The diagnostic tools available in the mid-1960s were primitive by today's standards - the chest X-ray, a few skin tests and lung function tests. The Abrams needle was available for pleural biopsies, but lung biopsies and most rigid bronchoscopies required a general anaesthetic. Over the next 20 years the situation was gradually transformed, fuelled by developments in respiratory physiology, bronchoscopy, biopsy techniques and radiology.

\section{Assessment of lung function}

Many of the techniques used to assess lung mechanics and airway function, from spirometry to plethysmography and flow volume curves, were available in more specialised centres in the 1960s and underpinned much clinical research. Equipment could be temperamental, however, and I spent many hours trying to repair concertina-type spirometers and replacing membranes on prototype blood gas electrodes. Peak flow meters, introduced by Martin Wright in the 1950s, were used increasingly to monitor asthma. The 1970s and 1980s saw the development of more robust equipment and more reliable results.

Investigation of breathing patterns during sleep led to the identification of sleep apnoea in the late 1970s, a major but previously unrecognised disorder. Diagnosis relied initially on polysomnography, a rather complex battery of tests, but simpler screening was introduced as pulse oximeters became available in the 1980s. Oximeters found a wide range of other uses, 
in acutely ill patients and in conjunction with simple tests such as the six minute walk test, developed to assess exercise ability in breathless patients. Improved methods to assess diaphragmatic and chest wall function were developed for patients with muscle weakness or chest wall problems, a previously rather neglected group of patients.

\section{Bronchoscopy and biopsy}

The introduction of fibreoptic bronchoscopy in the 1970s had a major impact on the practice of respiratory medicine, almost equivalent to that of the cardiac catheter. Chest physicians welcomed the opportunity to be involved more directly in diagnosis and management and in time overcame reservations from surgical colleagues. Bronchial biopsies could now be obtained in ill patients together with transbronchial biopsies and bronchoalveolar lavage, revolutionising the investigation of many conditions including lung cancer and diffuse lung diseases. A range of palliative procedures for endobronchial lesions were introduced later including laser therapy, brachytherapy and stenting while some earlier techniques, such as thoracoscopy, gained a new lease of life with advances in video imaging and biopsy techniques.

Not all new techniques, however, were successful. Using a percutaneous drill to biopsy the lung avoided the need for a general anaesthetic, but with levels of noise and vibration approaching those of a road drill it proved too stressful for both patient and doctor.

\section{Imaging techniques}

It is difficult to imagine practising respiratory medicine without computed tomography (CT) scans, but until the mid-1980s we relied on chest X-rays, tomograms and occasionally bronchograms. The uptake of CT scanners was initially frustratingly slow due to the high costs involved, and many hospital staff were engaged in fund-raising ventures. Once acquired, CT scans became indispensable, revolutionising the investigation of both discrete and widespread lung problems. Ultrasound and radionuclide scanning techniques have also made the investigation of many chest problems more accurate and safer.

\section{Other tools}

Patients with some of the rarer respiratory diseases have benefited from blood tests introduced since the 1970s, including alpha-1 anti-trypsin measurements and phenotype assays, genetic tests for CF, precipitating antibodies for allergic alveolitis and anti-cytoplasmic antibodies for various vasculitides.

\section{Discovering new diseases}

The recognition that several occupational lung diseases, such as farmer's lung, were all manifestations of an allergic alveolitis with precipitating antibodies, occurred during my training. This was important to recognise since although uncommon the condition is treatable and preventable. We enjoyed looking out for more bizarre causes such as paprika splitters lung. The adult respiratory distress syndrome was first described in 1967, but it took a decade or more to be widely accepted as a discrete entity rather than a manifestation of end-stage lung problems. It is surprising that a condition as common as sleep apnoea was not recognised in the 1960s. An occasional patient was diagnosed as having the Pickwickian syndrome but the crucial causative role of disordered breathing during sleep was not appreciated. Papers on sleep apnoea were published in the late 1970s and within five years the underlying physiological problem, the main features of the condition and an effective treatment (continuous positive airway pressure (CPAP)) had been described.

\section{Treatment and management}

Management has of course changed remarkably over the last 40 years. For example, the word tuberculosis still engendered great fear and a major challenge was to persuade patients that they would get better as long as they took treatment regularly, intramuscular streptomycin initially, plus large and indigestible tablets containing isoniazid and para-aminosalicylic acid daily for 18 months. Practices were slow to change and persuading patients, and sometimes hospital staff, that most patients did not require isolation or even hospital admission took time.

We also had to overcome prejudices about asthma - that it was not serious and that its origins were 'psychological'. The former was essentially put to rest by demonstrating the great variability in peak flow in patients in and out of hospital, and by the epidemic of asthma deaths in the 1960s. There was concern when the increasing death rates were attributed to excessive use of isoprenaline, since patients much preferred the metered-dose inhaler, available over the counter, to the cumbersome old squeeze-bulb inhalers (Fig 1). It was fortunate that salbutamol soon became available since alternative treatments for asthma were limited: desensitisation (controversial even then), theophyllines (often combined with ephedrine, a barbiturate or antihistamine in drugs such as franol), occasionally prednisone or subcutaneous adrenaline. Sedatives, or an evening whisky or brandy, were even given cautious recommendations in textbooks. The lack of effective prophylactic treatments apart from oral corticosteroids led to various experimental but short-lived treatments such as vagotomy and bronchoalveolar lavage.

There was much interest in ventilatory failure when I trained since carbon dioxide tensions could be measured and work by Campbell and others had shown the dangers of uncontrolled oxygen. Ventimasks were available and nikethamide was given to stimulate breathing, with the added bonus of waking the patient up enabling them to cough. Positive pressure ventilation was just starting and was sometimes carried out on general wards prior to intensive care units. Relatively simple, pressure-cycled ventilators had been developed in response to a recent polio epidemic where a hand ventilation by relays of students proved to be a most effective, though labour intensive, method of reducing the acute mortality.

Since the 1960s several new respiratory drugs have appeared 
although not many new classes of drugs compared with other specialties. Rifampicin and to a lesser extent ethambutol had a major impact on mycobacterial diseases and the increased choice of broad spectrum antibiotics has prolonged the lives of many patients, particularly those with suppurative lung conditions such as CF. Chemotherapy for lung cancer has improved in terms of tolerability but has been less effective in prolonging life than had been hoped. Modifications to isoprenaline and cortisone have produced drugs which have had the greatest impact on morbidity from asthma, and to a lesser extent COPD - beta ${ }_{2}$ selective drugs in the late 1960s, long-acting beta ${ }_{2}$-agonists in the 1990s and, most important, inhaled steroids in the 1970s. The leukotriene modifying drugs and anti-IgE monoclonal antibodies are new classes of drugs but their impact has been small to date.

For most respiratory conditions rationalising management on the basis of findings from clinical trials has made at least as large a contribution to improving patient outcomes as the introduction of new drugs. The increased life expectancy for patients with CF is due to careful management of all aspects of the condition rather than any specific drug treatment. Wide acceptance of clear guidelines for managing TB, based on solid evidence from large-scale pragmatic trials, accounts for the relatively low incidence of drug-resistant TB in the UK today. The asthma death epidemics focused attention on asthma management and stimulated the production of management guidelines in the UK in 1990. They were effective because they focused on a few simple messages, and were widely disseminated. Guidelines are now available for almost all respiratory conditions, though often less evidence based.

Certain groups of patients have been helped by the development of physical methods to assist breathing, particularly at night. There was incredulity initially at the idea that patients with

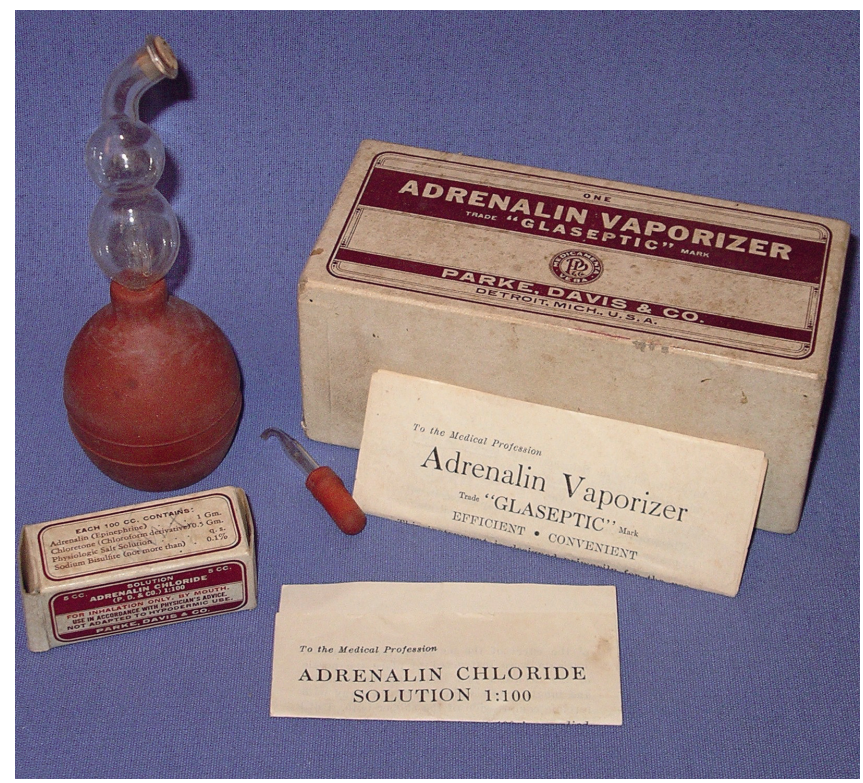

Fig 1. Treatment for asthma in the mid-20th century. Courtesy of Mark Sanders and www.inhalatorium.com. sleep apnoea might actually sleep better and feel more refreshed in the morning if they wore a nasal mask attached to a machine delivering CPAP overnight. But clearly this was the case and domiciliary use of CPAP like non-invasive ventilation for patients with muscle weakness can improve quality of life immeasurably; both techniques also have a role in high dependency units and intensive care.

Much respiratory disease can be attributed to ambient and occupational air pollution, including cigarette smoke, and following the smogs in the 1950s the UK has remained at the forefront of research to quantify these effects and set air quality standards. Implementation has been patchy however and while the incidence of some diseases, such as coal miner's pneumoconiosis, has fallen dramatically, slow implementation of asbestos standards means that the incidence of mesothelioma will continue to rise until at least 2010.

Progress in reducing cigarette smoking has also been patchy. The dangers of smoking were recognised when I trained though only a few pioneers such as Keith Ball at Central Middlesex Hospital ran anti-smoking clinics. In the 1980s it was not unusual for doctors to smoke and chest specialists with a cigarette packet prominently displayed in a shirt pocket could still be found.

\section{Information availability}

Textbooks and libraries assumed greater importance before the internet and proliferation of guidelines, and the Index Medicus and Royal Society of Medicine library were valuable resources for my early research. The 1960s saw the publication of several authoritative and influential books. Lung function edited by Cotes became indispensable for lung function laboratories; ${ }^{1}$ Ventilation: blood flow and gas exchange by West was essential reading for anyone struggling with ventilation-perfusion mismatching; ${ }^{2}$ Respiratory failure by Sykes, McNicol and Campbell provided practical help for a condition that was difficult to manage; ${ }^{3}$ and Crofton and Douglas's respiratory diseases provided a comprehensive textbook covering all aspects of respiratory medicine. ${ }^{4}$ The latter in particular gave physicians the information and confidence to treat patients with rarer lung diseases, rather than refer them for a second opinion. Prescribing was a rather vague art in the 1960s and anecdotes about idiosyncratic prescribing by consultants enlivened meal times as a junior doctor (no clinical governance then). A major revamp of the British national formulary (BNF) in 1980 was immensely helpful; a small hard backed book detailing ancient drugs dispensed in grains, minims and tinctures metamorphosed almost overnight into the modern BNF. ${ }^{5}$

\section{Respiratory medicine as a profession}

When I trained respiratory medicine had two professional societies, the British Thoracic Association, arising from the British Tuberculosis Association, and the Thoracic Society, which tended to represent the younger, more physiologically orientated chest physicians. The two societies merged to form 
the British Thoracic Society (BTS) in 1982, reflecting the gradual melding together of these two historical strands of the profession. The BTS has played a major role in developing the specialty over the last 25 years and led the way in many areas including clinical governance, training, and lobbying for smoking cessation services and better services for patients.

In the 1960s, today's manpower figures for respiratory medicine were unimaginable. There were around eight respiratory senior registrars in the country, compared to 275 specialist registrars now, and far fewer chest consultants. Many consultants in district general hospitals were single-handed until recently, despite extremely high workloads. The four UK chairs in respiratory medicine in 1984 have now expanded at least tenfold.

\section{Back to the future}

I entered respiratory medicine at an exciting time when expectations were high. Many of these expectations have been fulfilled, in part at least. Several conditions can be, and are, prevented, some are cured and most patients with chronic respiratory conditions can expect a better quality of life. There have also been some major disappointments. The eradication of TB was in sight in the 1980s but due to AIDS and multi-drug resistance, it is now a bigger problem than ever worldwide. The fiveyear mortality for lung cancer has improved little despite progress in diagnosis and treatment, and the reduction in particulate air pollution has had less effect on the incidence of COPD than was hoped.

The factors underpinning the successes during the last 40 years are various and include individual innovators and inventers, new drugs from the pharmaceutical industry, leadership in ensuring that good practices are widespread and a strong track record of research. The UK has played a major role in clinical, epidemiological and physiological research throughout my career, much of it pragmatic and feeding through directly to patient care. The increase in research in cell and molecular biology and clinical genetics, in conjunction with good clinical research, holds the promise of more fundamental benefits for patients in the future.

\section{Acknowledgements}

Comments by Dewi Davies and Martin McNicol on an early draft were extremely helpful, and in the latter case reminiscent of comments made 40 years ago.

\section{References}

1 Cotes JE. Lung function: assessment and application in medicine. Oxford: Blackwell Scientific Publications, 1965.

2 West JB. Respiratory physiology - the essentials. Oxford: Blackwell Scientific Publications, 1974.

3 Sykes MK, McNicol MW, Moran Campbell EJ. Respiratory failure. Oxford: Blackwell Scientific, 1969.

4 Crofton J, Douglas A. Respiratory diseases. Oxford: Blackwell Scientific Publications, 1969.

5 British Medical Association and Royal Pharmaceutical Society of Great
Britain. British national formulary. London: BMJ and Royal Pharmaceutical Society Publishing, 2007. www.bnf.org/bnf/bnf/current/index.htm

\section{CURRENT KEY DEVELOPMENTS}

\section{Cystic fibrosis}

\author{
Duncan Geddes MD FRCP \\ Professor of Respiratory Medicine, Royal Brompton National Heart \\ and Lung Hospital
}

Email: d.geddes@rbh.nthames.nhs.uk

\section{Background}

By the end of the 1960s cystic fibrosis (CF) was established as a rare and fatal disease of childhood defined by recessive inheritance, pancreatic insufficiency, lung infections and a positive sweat test. Since so few children with CF lived past their teens the associated diabetes, liver disease and male infertility attracted little attention. Scientists avoided CF as it was poorly funded, poorly understood and advances seemed unlikely. Nevertheless, shining through this gloom were rare beacons of optimism, a few enthusiastic paediatricians had given aggressive treatment with dietary supplements and antibiotics and shown that CF need not always progress rapidly to death.

The first adult CF clinic in the UK started at the Brompton Hospital in 1966. New pancreatic enzyme formulations, new antipseudomonal antibiotics, and physiotherapy techniques led to a shift in attitudes. More importantly CF families set up national bodies (Cystic Fibrosis Research Trust in the UK and Cystic Fibrosis Foundation in the USA) to raise money for research, educate CF parents and press for higher standards of care. Interested clinicians were converted into CF specialists, attendance at a CF centre became the norm and scientists were attracted into the field by the new money and astonishing advances in molecular genetics. Specialising in CF became fashionable and meetings, previously sad and poorly attended, began to buzz. Progress, excitement and optimism were in the air and the journals were full of advances: neonatal screening, prenatal diagnosis, high fat diets with acid resistant pancreatic enzymes, regular intravenous (iv) and nebulised antibiotics, all cascaded into print. The scientists joined in and after a few false starts - serum CF factors and spurious immune defects progress was rapid: defective ion transport linked the sweat gland to the lungs and pancreas (1981) and the new techniques of chromosome walking and jumping helped identify the $\mathrm{CF}$ 